\title{
Trajectory based Recovery of Index Finger Articulated Pose during Palmar Grasp
}

\author{
Avik Chatterjee \\ CSIR-CMERI, \\ M.G.Avenue, \\ Durgapur, India.
}

\author{
A. Mahapatra \\ CSIR-CMERI, \\ M.G.Avenue, \\ Durgapur, India.
}

\author{
S. Majumder \\ CSIR-CMERI, \\ M.G.Avenue, \\ Durgapur, India.
}

\author{
I Basak \\ NIT Durgapur, \\ M.G.Avenue, \\ Durgapur, India.
}

\begin{abstract}
This paper describes our experimental and analytical study of recovering index finger pose from tip trajectory during palmar grasp. Our study experimentally evaluates a kinematical model that can be used to reduce the number of surface markers in each finger for motion estimation and its segmental kinematics. We captured the trajectory of the index finger tip and joint angles in typical fist closing mode (palmar grasp), based on the concept of planar homology in projective space and then investigated the inverse kinematics solutions for the correlation. Jacobian based Damped Least Square (DLS) with variable damping parameter $\lambda$ has been implemented. The DLS method, though iterative, shows reasonably fast convergence with in 3-10 iterations in feed forward mode and has better concurrence with the experimental values in recovery of articulated pose.
\end{abstract}

\section{General Terms}

Robotics, Computer Vision.

\section{Keywords}

Index Finger, Damped Least Square (DLS), Jacobian, Planar Homology, Inverse Kinematics

\section{INTRODUCTION}

We ask that authors follow some simple guidelines. In essence, we ask you to make your paper look exactly like this document. The easiest way to do this is simply to download the template, and replace the content with your own material. In the recent years, the interest in hand motion assessment has been increased due to research in the field of Human Computer Interaction (HCI), Man Machine Interaction (MMI), Ergonomics, Robotics and Biomechanics. The kinematics of human hand motion has been mostly studied with the common assumption of Distal Interphalangeal Joint (DIP) and Proximal Interphalangeal Joint (PIP) dependency, to reduce the problem complexity and to avoid redundancy [1][2]. To solve the nonlinear equations, various methods like binary search, finger tip distance and slant angle, pseudo inverse and iterative optimization techniques were adopted. Subsequently other approaches based on vision, appearance and machine learning were gradually introduced.

In vision based hand gesture recognition system, the recovery of articulated hand pose from images is often formulated as a tracking problem [3]. Hand shape models are fitted with statistical methods such as local principal component analysis [4], sequential Monte Carlo [5] and kinematic models with articulation [6]. More recent efforts have reformulated the problem within a Bayesian framework [7] although; it is a computationally expensive approach.
Appearance based approaches estimate hand states directly from images after learning the mapping from the image feature space to the hand configuration space. These approaches are often based on skin color regions in the image [8], motion capture through machine learning [9], multiple surface markers with multiple camera setup [10] or using inertial navigation sensors (INS) along with resistive bend sensors. In machine learning approach, prior knowledge about human motion, is to be learnt from large training data set, to resolve ambiguities which is not trivial.

The primary challenges of computer vision based approaches are self occlusion among fingers during full fist closure and hence involve multiple markers fixing in each finger along with multiple camera for mapping between marker motion and respective joint motion. Multiplicity of surface markers is prohibitive during palmar grasp. It not only acts as a barrier in free motion, as the markers are to be put near the joints, but also highly susceptible to slip, as the skin is stretched considerably during full fist closure due to the fact that joints are stretched to their natural limits and the fingers curled with full 270 degree sweep of the tip segment. The INS sensing approach for grasping motion sensing makes the hand bulky for attaching the various sensing elements along with their signal processing and data transmission electronics and thereafter makes the motion unnatural and restrictive, particularly during full fist closure (palmar grasp).

This provides motivation to investigate a kinematical model which can take its input from the trajectory of a single marker fixed at finger tip and resolve the joint angles (articulated pose) during palmar grasp. This particular problem has not yet been fully addressed.

The paper is organized as follows. In section 1, the forward kinematics of index finger with its joint angles nomenclature has been discussed. In section 2 , we have formulated its inverse kinematics. The experimental framework has been discussed in section 3 . In section 4 , we have discussed the experimental results and the performance of our methodology. Finally, section 5 draws some conclusions and gives directions for future work.

\section{FORWARD KINEMATICS (FK)}

The index finger has 4 degree of freedom (DOF), 2 DOF for Metacarpo Phalangeal Joint $\left(M C P: \theta^{1}=\theta_{M C P}^{Y}\right)$, $\left(M C P: \theta^{2}=\theta_{M C P}^{Z}\right), \quad 1 \quad$ DOF each for Proximal Interphalangeal Joint $\left(P I P: \theta^{3}=\theta_{P I P}^{Z}\right)$ and Distal Interphalangeal Joint $\left(D I P: \theta^{4}=\theta_{D I P}^{Z}\right)$. Coordinate frames as per Denavit-Hartenberg notation $(\mathrm{D}-\mathrm{H})$ were attached to the finger joints as shown in Figure 1, resulting the finger tip 
transformation by the matrix $A=R_{z} \theta_{i} * T_{z} d_{i} * T_{x} a_{i-1} * R_{x} \alpha_{i-1}$ where a and $\mathrm{d}$ are translations along $\mathrm{X}$ and $\mathrm{Z}$ axes respectively and $\alpha, \theta$ are rotations along $X$ and $Z$ axes respectively. Joint rotation along $\mathrm{Y}$ axis is the abduction-adduction ( $\mathrm{ab} / \mathrm{ad})$ motion and along $\mathrm{Z}$ axis is the flexion-extension (f/e) motion of the joint. The length of proximal phalange, intermediate phalange and distal phalange are 11,12 and 13 respectively which are also referred as joint offsets as per D-H standard convention. Given the joint angles $\theta^{i}(i=1 \ldots 4)$ and length offsets $\left(l^{i}: i=1 . .3\right)$ the FK formulation results in finger tip trajectory position and orientation as $x=[p \phi]^{T}=f\left(\theta^{i}\right)$ with respect to the base frame (wrist) and with the assumption of no joint dislocation, where $\mathrm{p}$ and $\phi$ are [1x3] position and orientation vectors respectively. The global roll-pitch-yaw motion of the hand is governed by the wrist. The joint parameters $\left(\theta^{i}, l^{i}\right)$ along with tip position and orientation

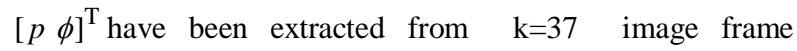
sequences during palmar grasp operation, but still the analytical form of $x=f\left(\theta^{i}\right)$ is required for the calculation of the Jacobian (J) during pose recovery.

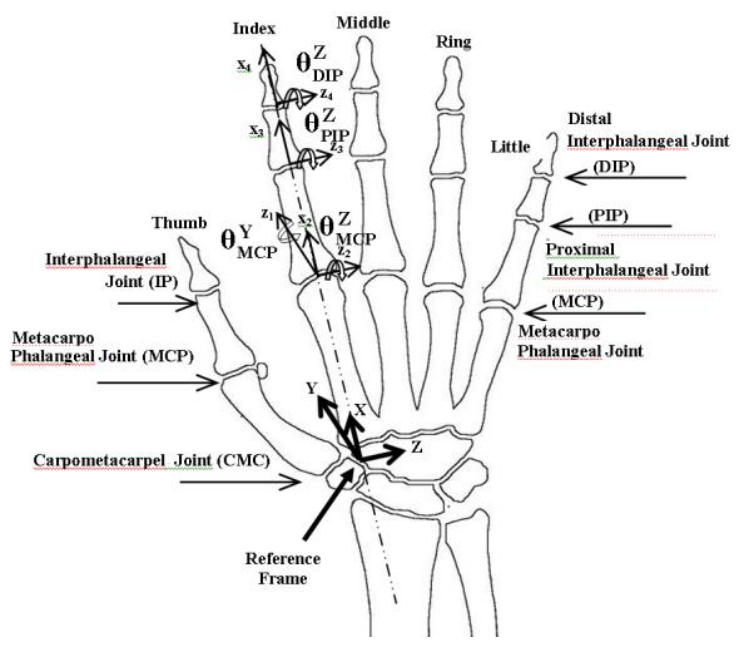

Figure 1 : Joint location and nomenclature [11].We used the indicated nomenclature hereafter.

\section{INVERSE KINEMATICS (IK)}

The forward kinematics relation is non linear and transcendental with redundancy of degree one, as the task space $x \in R^{m} \rightarrow m=3$ and the joint space is $\theta \in R^{n} \rightarrow n=4$. The differential changes in $\theta$, is related to differential changes in $x$ as $d x=J(\theta) d \theta$, where $J(\theta)$ is the geometric Jacobian matrix [Appendix-A1]. Since $d \theta=J(\theta)^{-1} d x$ and $J(\theta)$ is non square, it is not directly invertible. Also singularities cause rank of the Jacobian to change. The Moore Penrose inverse $\left(\mathbf{J}^{\dagger}\right)$ of $J(\theta)$ also commonly called pseudo inverse, has been widely used in IK to handle redundancy $\left(\Delta \theta=J^{\dagger} \Delta x\right)$. It performs poorly because of its instability near singularities. Hence the inverse solution of this problem has been formulated using DLS method which has better behavior near the singularities through Levenberg-Marquardt minimization. The method was first applied to solve IK by Wampler [12], Nakamura and Hanafusa [13]. The implementation of DLS method [14] is mentioned in Appendix-A3.

DLS solution takes into account both the accuracy and norm of the solution at the same time, where $\lambda$ is used to specify the relative importance of the norms of joint angles and the tracking accuracy. Moreover, if the singular values $\left(\sigma_{i}\right)$ are much larger than the damping factor (which is likely to be true when the solution is far from singularities), then there is little difference between pseudo inverse and DLS, since in this case $\frac{\sigma_{i}}{\sigma_{i}{ }^{2}+\lambda_{i}^{2}} \approx \frac{1}{\sigma_{i}}$. On the other hand, if the singular values are of the order of (or smaller) the damping factor (which is likely to be true when the solution is near to singularities) then the denominator tends to reduce the potentially high norm joint values. The practical significance of this method is that it gives a unique solution which most closely approximates the desired joint space value among all possible joint values. Joint Limit Avoidance (JLA) is implemented by

$\lambda_{\mathrm{i}}=\Phi(\theta)=\eta\left[\frac{2 \theta_{i}-\theta_{i \max }-\theta_{i \min }}{\Delta \theta}\right]^{\mathrm{p}}$

for $\quad \theta_{i \text { min }} \leq \theta_{i} \leq \theta_{i \max }, \quad \eta=1, \mathrm{p}=6$ (even) and $\Delta \theta=\left(\theta_{i \max }-\theta_{i \min }\right)$ for $\mathrm{i}^{\text {th }}$ joint. When $\theta_{i}$ exceeds the joint limit, $\lambda_{i}$ becomes high and tries to bring back the solution within the feasible range. If $\theta_{i}$ is within the limit, $\lambda_{i}$ is low and the solution converges within feasible range. The larger value of $\mathrm{p}$ is suggested to make the base of the function $\Phi(\theta)$ flatter and to ensure rapid increase of $\lambda_{i}$ in the event $\theta_{i}$ exceeds the joint limits.

The IK of the model has been solved by conventional analytical method considering DIP-PIP dependency, and by DLS method, without considering any joint dependency. A fixed value of $\lambda_{5}=\lambda_{6}=0.7$ has been used instead of a computed one as we have 4 joint variables making the damping factor a $6 \times 6$ diagonal matrix $\left(\lambda_{\mathrm{D}}\right)$. In the first frame the solution starts at qi $=\left[\begin{array}{lll}0 & \mathrm{pi} / 2 & 0\end{array}\right]$ and converge rapidly into target position in 33 iterations. The initial joint angle vector of the current frame is the solution set of the previous frame ( $q_{i}^{0}=q_{i-1}^{l}$, where $l$ is the last iteration result of $q_{i-1}$ frame). This feed forward algorithm helps in rapid convergence of solutions in each frame within 3 to 10 iterations, as the norm of the incremental angle vector (dq) approaches to the value of iteration termination tolerance of value of $1 \mathrm{E}-06$.

\section{EXPERIMENTAL FRAMEWORK}

To compare the recovered pose $\left(q^{i} \forall i=1 \ldots 4\right)$ with the measured joint angles of index finger $\left(\theta^{i} \forall i=1 \ldots 4\right)$, fist closing operation has been recorded for 5 different individuals by both digital cameras in a canonical stereo experimental setup (Figure 2). For each individual, 5 cycles are recorded. The mean of MCP ab/ad ( $\left.\theta^{1}\right)$ has been assumed zero during experimental recording and assigned a small positive value of 0.1 in the formulation to avoid ill conditioning during SVD in iteration. This assumption has been used since the range of motion is limited ( \pm 15 degree) [3] and it simplifies the measurement complexities from $3 \mathrm{D}$ to $2 \mathrm{D}$. The average recorded time period for 1 complete closing and opening 
cycle is $4 \mathrm{~s}$ (slow grasp). There is variation in pace for different subjects and for different cycles of same subject but the images are recorded when their pace stabilized to $4 \mathrm{~s}$ avg.

For extraction of $\theta^{i}$, the image sequence of left camera is used as it provides the approximated fronto-parallel view along with the positions of the markers (marked by black dots as shown in Figure 2).

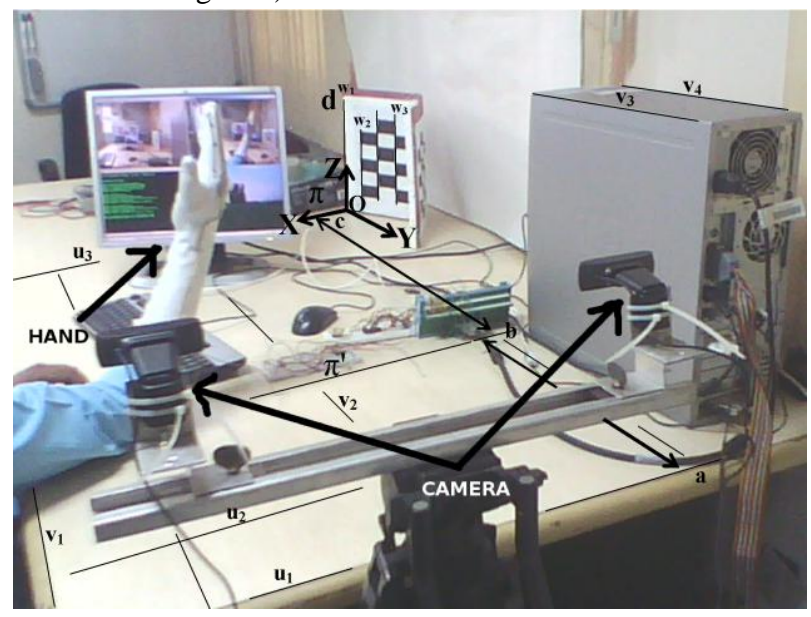

Figure 2 : Canonical stereo experimental setup

The closing cycles are separated for study and 37 image frames of interest are extracted for each closing cycle. The images are corrected for lens distortion [15], and converted into grayscale followed by edge extraction (Canny's method) with a threshold value range of $0.2-0.5$ and sigma of the Gaussian filter as 1. Edge extraction is followed by line fitting among the edges with a maximum deviation from original edge of 2 pixels. Fitted line segments are then traversed to select the region of interests namely the baseline regions B1 and $\mathrm{B} 2$ and four joint regions viz. MCP joint region (A1), PIP joint region (A2), DIP joint region (A3) and finger tip region (A4) as shown in Figure-3 for the $7^{\text {th }}$ frame (case-1).

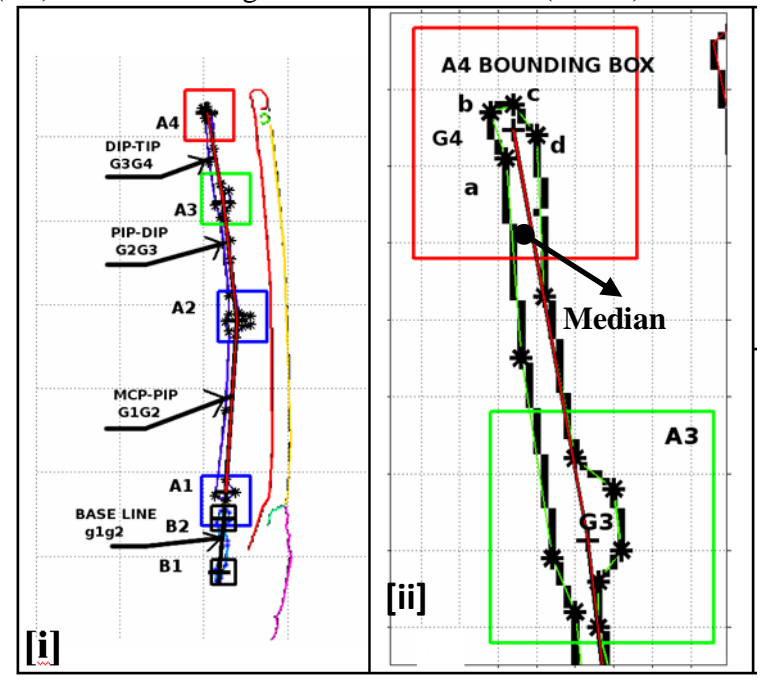

Figure 3 [i] Regions B1, B2 and A1-A4 for 7th frame. [ii] $A 4$ and $A 3$ region details. G4 and $G 3$ are the centroids. G3G4 is the DIP-TIP segment (median line).

Each region (B1, B2, and $\mathrm{A} 1$ to $\mathrm{A} 4$ ) is represented by a bounding box having a convex polygonal area inside defined by the intersection points of fitted line segments within the box. The centroid of each convex polygonal region represents the end point of the median line.

Once the centroids have been extracted, distances and angles between centroids in a particular frame, have been estimated based on the work of single view metrology by Criminisi [16]. The reference plane is $\pi(\mathrm{XZ})$ and measurement plane is $\pi$ ' (Figure 2). The affine axis is XYZ. The reference known height is od, in plane $\pi$ and other reference known distances are $\mathrm{cb}$ and $\mathrm{ba}$. The line segments used for extraction of vanishing points in $\mathrm{X}, \mathrm{Y}$ and $\mathrm{Z}$ directions are ( $\mathrm{u} 1, \mathrm{u} 2, \mathrm{u} 3)$, (v1, v2 , v3, v4) and (w1, w2, w3). Thick black lines are drawn in the figure to clearly identify the orthogonal line segments used for estimation of vanishing points $\mathrm{u}, \mathrm{v}$ and $\mathrm{w}$ in the $\mathrm{X}, \mathrm{Y}$ and $\mathrm{Z}$ directions. The distance measuring formula is mentioned in Appendix-A2. The angles have been estimated from tangent formulae as we have extracted the centroid coordinates in Euclidian space by applying planar homology on projective space [16].

The data acquired from the image frames are tip position and orientation ( $p, \phi)$, joint angle vector $\theta^{i}: \forall i=1 \ldots 4,\left(\theta^{1}=0\right)$, which is the angular distance between the line segments, namely base line segment, MCP-PIP segment (11), PIP-DIP (12) segment and DIP-TIP (13) segment and the lengths of $l 1, l 2$ and $l 3$. The median line segments and extracted joint angles are superimposed on the original images of 7 th and 37 th frames to show the agreement in Figure-4.

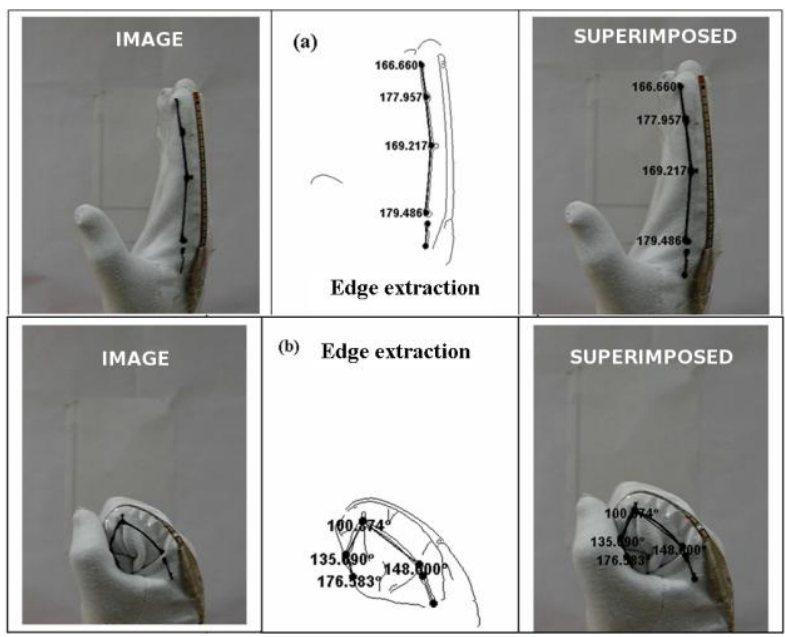

Figure 4 : Extraction of joint angles along with length offsets for subject-1, cycle -1.(a) 7th frame (b) 37th frame. Joint angles are superimposed on image.

\section{RESULTS AND DISCUSSION}

The value of joint angles obtained form DLS method and from analytical solution have been separately plotted for 5 different subjects in Figure 5. The activation of Joint Limit Avoidance scheme, implemented by variable damping factor $(\lambda)$ for each joint angle is noticeable in DLS solution of $\operatorname{DIP}(\mathrm{f} / \mathrm{e})$ joint angles $(\theta 4)$ for all cases, when it goes out of minimum bound of $0^{\circ}$. The DLS solution of MCP ab/ad ( $\left.\theta 1\right)$ is almost zero as per the assumption. The MCP-f/e is low during initial $0-15$ frames, which corresponds to nearly $1 / 3$ rd of the total closing cycle of $2 \mathrm{~s}(2 \mathrm{~s} \sim 37$ frames). Afterwards it shows a steep rise and reaches the joint limit. This behaviour is noticed in all cases. On the contrary the DIP-f/e $((\theta 3)$, is active from the start of the cycle and shows approximately linear rising behaviour up to its joint limit. The 

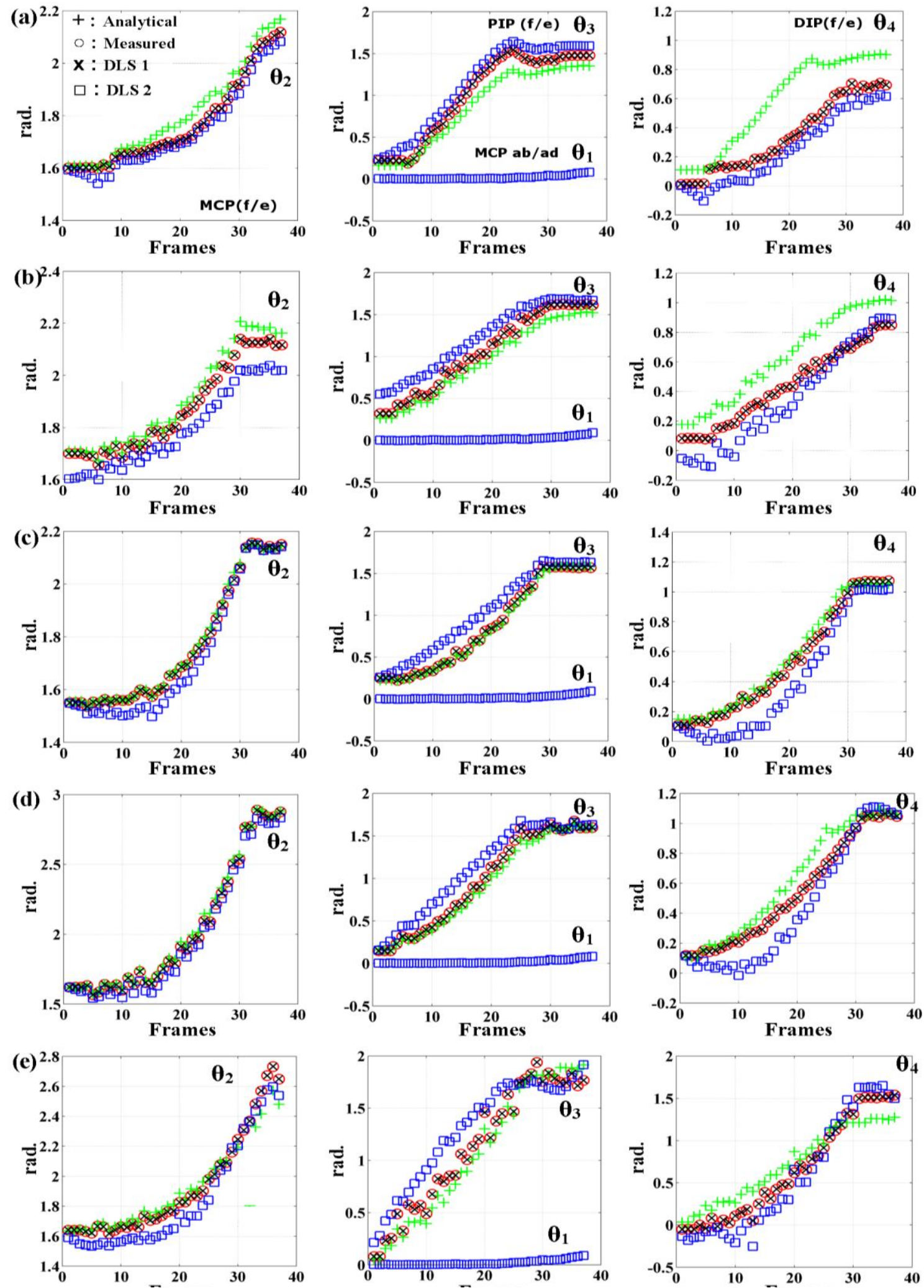

Figure 5 : Analytical and Jacobian Solutions (DLS) of joint angles compared with experimental values. (a) Subject-1 (b) Subject-2 (c) Subject-3 (d) Subject-4 (e) Subject-5. In DLS-1 the differential motion vector (de) is obtained from forward kinematics transform $(A i=f(l i, \theta i))$ of current joint angle $\theta i$, starting from initial solution [ 0 pi/2 0 o] and forward kinematics transform $(A m=f(l i, \theta m))$ of measured angles as goal in each iteration. Hence it nearly coincides with the measured angles. It has been done to theoretically test the DLS scheme. In DLS-2, de is obtained from forward kinematics transform (Ai) of current joint angle $\theta i$, starting from same initial solution and measured position and orientation of the finger tip as goal in each iteration, which is the actual result. 

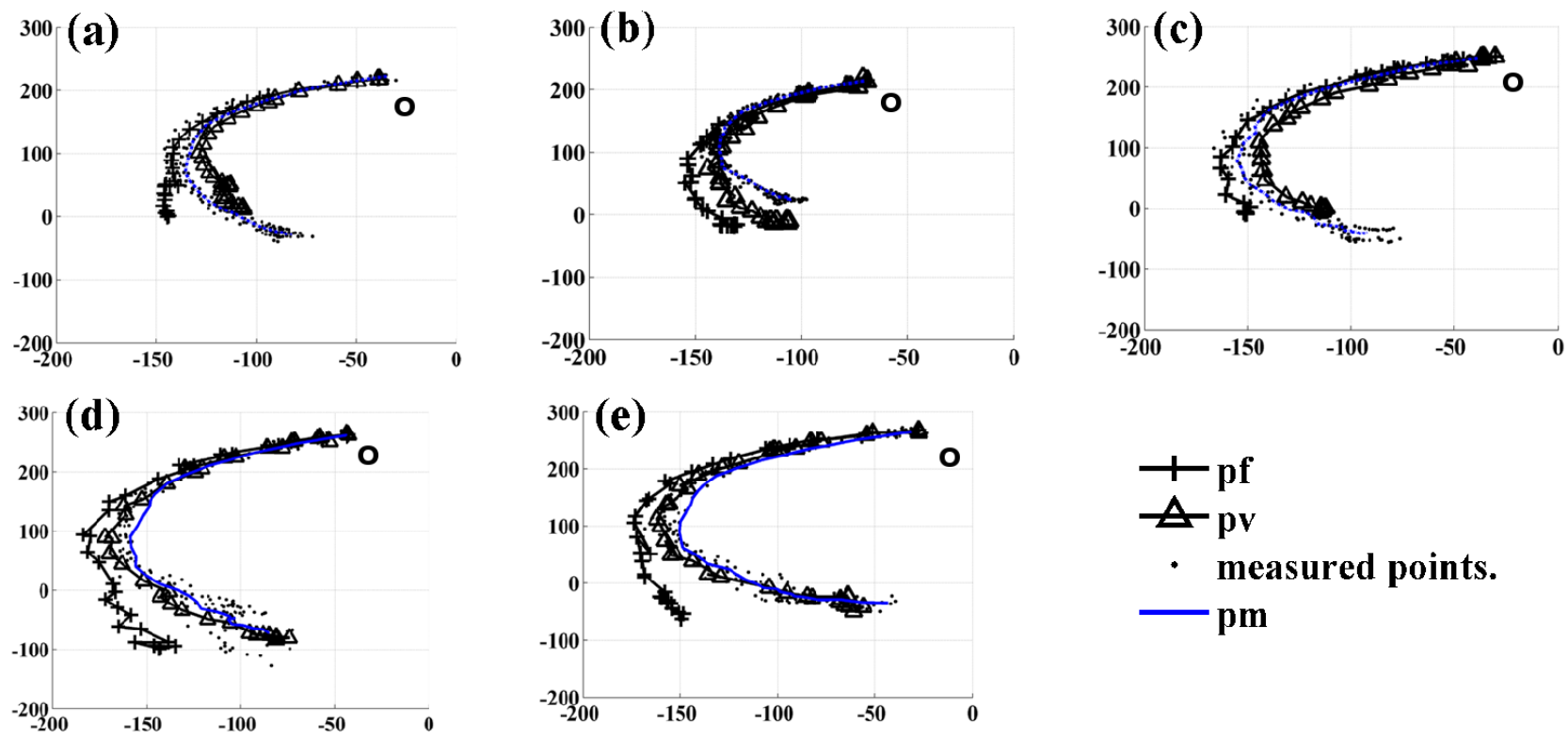

Figure 6 : Finger tip trajectory for 5 different subjects. (a) Subject-1 (b) Subject-2 (c) Subject-3 (d) Subject-4 (e) Subject-5. X and $Y$ axis in pixel units. The measured tip position mean curve $\left(p_{m}\right)$ and forward kinematics with measured length offsets curve $\left(p_{v}\right)$ are translated to coincide with the starting point $(o)$ of forward kinematic solution $\left(p_{f}\right)$, so that curves can be compared and translation is shape and size invariant.
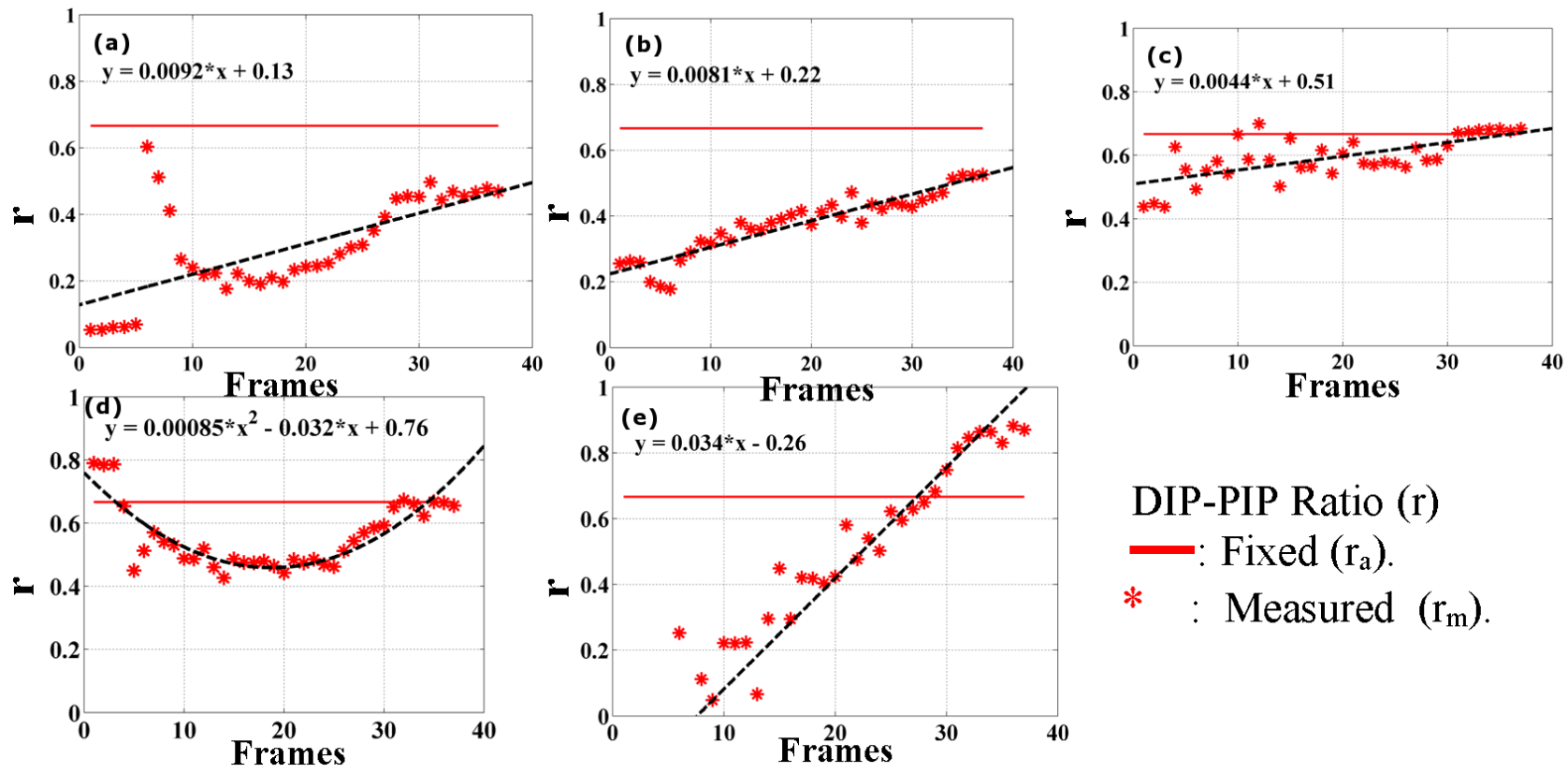

Figure 7: DIP-PIP ratio of measured data for each observation. (a) Subject-1 (b) Subject-2 (c) Subject-3 (d) Subject-4 (e) Subject-5.

measured trajectory $\left(\mathrm{p}_{\mathrm{m}}\right)$ of the finger tip, extracted from image processing, have been plotted separately for 5 different subjects as shown in Figure 6. The shape of the motion path is similar in all cases. The forward kinematic solution with fixed joint offsets (curve- $\mathrm{p}_{\mathrm{f}}$ ) and measured joint offset (curve$p_{v}$ ) have also been plotted to bring out a graphical comparison. Curve $\mathrm{p}_{\mathrm{f}}$ shows agreement with mean curve $\left(\mathrm{p}_{\mathrm{m}}\right)$ up to its left convex peak and thereafter it shows nonconformity. The left convex peak of the actual tip trajectory curve is the position where PIP -f/e $\left(\theta^{3}\right)$ and DIP- f/e $\left(\theta^{4}\right)$ are at their limits and MCP (f/e) joint angle is half way $\left(\sim 45^{\circ}\right)$. After that as the fist closes completely, the joint offsets are stretched to maximum, which the forward kinematics solution with fixed joint offset can not reflect. This is reflected in the forward kinematics solution with measured joint offsets, where the curve at least follows the actual tip trajectory along with its bend up to the last frame.

The DIP-PIP ratio for the measured data (Figure 7) for each observation shows a linear approximation in 4 out of 5 cases. 
For subject-4, the relation is better approximated by a quadratic function. In the conventional analytical approach, which considers fixed DIP-PIP dependency, it has been observed that, this does not hold good for all individuals in full fist closure and the DIP-PIP ratio is not constant for the entire range of motion.

\section{CONCLUSIONS}

We have developed full 4 DOF kinematical model of index finger with its IK solved by DLS method, without considering any joint dependency. The problem is also solved by conventional analytical method, considering DIP-PIP dependency. The solution set is validated against the experimental data of joint positions extracted through image processing for full fist closure (palmar grasp). The conventional analytical approach with joint dependency does not hold good for all individuals in full fist closure (Figure 5), particularly near full closing ranges and the DIP-PIP ratio is not constant for the entire range of motion (Figure 7). The DLS formulation results in fast convergence in feed forward mode (within 3 to 10 iteration per frame) and its results conform with experimental joint angles, during full fist closure, when the finger tip trajectory is defined as a goal.

Results of this experiment, project the DLS method as one of the promising candidate for modeling human palmar grasp motion in real time given finger tip trajectory. Of course it is an iterative method which has its own overhead, but at the same time it drastically reduce the number of markers from 5 per finger (1 MCP ab/ad + $1 \mathrm{MCP}$ f/e + $01 \mathrm{PIP}$ f/e + $1 \mathrm{DIP}$ f/e +1 tip marker) to 2, i.e. 1 for $\mathrm{MCP}$ ab/ad and 1 for finger tip, if actual MCP ab/ad motion $\left(-15^{\circ} \leq \theta 1 \leq 15^{\circ}\right)$ needs to be captured. This directly reduces the image processing overhead.

It is beyond any doubt that measurement by putting multiple surface markers or motion sensors on each joint will give more precise values but all these make the natural motion of fist closing restrictive due to their bulkiness (inertia sensors) or slippage (surface markers) in full closed condition of fist, due to high stretching of skin during palmar grasp.

This approach also gives a direction towards solving the problem of self-occlusion of fingers in fist closing operation which is one of the major hurdles in pure image based tracking approach, as we have to track only the finger tip, retaining full DOF of human hand and without any joint motion dependency of conventional methods.

The experiment also reveals that the nature of the finger tip trajectory in fist closing operations for different people is similar in shape (Figure 6) with minor variations, depending on phalange lengths. This attribute can be further investigated for its feasibility in qualitative detection of imperfections in grasping motion for persons having impaired finger movement in the future scope of work.

\section{ACKNOWLEDGEMENTS}

The authors would like to thank Prof. Gautam Biswas, director of Central Mechanical Engineering Research Institute (CMERI), constituent establishment of Council of Scientific and Industrial Research (CSIR), New Delhi, for extending the facilities and infrastructure for carrying out experiments.

\section{REFERENCES}

[1] J. Lee and T.L. Kunii.1993. Constrained Based Hand Animation. Models and Techniques in Computer Animation. Springer. Tokyo. pp 110-127.
[2] John Lin, Ying $\mathrm{Wu}$ and Thomas S. Huang. 2000. Modeling the constraints of human hand motion, Proceedings of Workshop on Human Motion, ISBN: 07695-0939-8, pp-121 - 126.

[3] S. Chen, Y. Li, and N. M. Kwok, 2011. Active vision in robotic systems: a survey of recent developments, International Journal of Robotics Research, vol. 30, no. 11, pp. 1343-1377.

[4] C. B. Phillips, J. Zhao, N. I. Badler. 1990. Interactive real-time articulated figure manipulation using multiple kinematic constraints. Proceedings of the ACM SIGGRAPH. pp 245-250.

[5] Michael Isard and Andrew Blake. 1998. CONDENSATION - conditional density propagation for visual tracking. International Journal of Computer Vision, 29:5-28,

[6] Y.Wu, John Lin, and T. S. Huang. 2001. Capturing natural hand articulation. In Proc. of International Conference on Computer Vision, pp: 426-432. Vancouver.

[7] B. Stenger, A. Thayananthan, P.H.S. Torr, R. Cipolla. 2006. Model-based hand tracking using a hierarchical Bayesian filter. IEEE transactions on pattern analysis and machine intelligence. 28(9):1372-1384.

[8] B. Stenger. 2006. Template based Hand Pose recognition using multiple cues. Proc. 7th Asian Conference on Computer Vision: ACCV 2006. pp: 551-560. Hyderabad. India

[9] V. Athitsos and S. Sclaroff. 2003. Estimating 3D Hand Pose from a Cluttered Image. Proc. IEEE Conf. Computer Vision and Pattern Recognition, vol. II, pp. 432-439.

[10] P. Cerveri, E. De- momi, N. Lopomo, G. Baud-bovy, R. M. L. Barros, and G. Ferrigno. 2002. Finger Kinematic Modeling and Real-Time Hand Motion Estimation, Annals of Biomedical Engineering, Vol. 35(11): 19892002.

[11] www.infovisual.info/03/ /027_en.html.

[12] C. W. Wampler and L. J. Leifer. 1998. Applications of damped least-squares methods to resolved-rate and resolved-acceleration control of manipulators, Journal of Dynamic Systems, Measurement and Control. 110: 3138.

[13] Y. Nakamura and H. Hanafusa. 1986. Inverse kinematics solutions with singularity robustness for robot manipulator control. Journal of Dynamic Systems, Measurement, and Control. vol 108, pp 163-171.

[14] Samuel R. Buss and Jin-Su Kim. 2005. Selectively Damped Least Squares for Inverse Kinematics. Journal of Graphics Tools. vol 10(3), pp 37-49.

[15] F. Devernay and O. Faugeras, 1995, Automatic calibration and removal of distortion from scenes of structured environments, In SPIE San Diego, CA, 1995, vol 2567.

[16] A.Criminisi, I. Reid and A. Zisserman, 2000, Single view metrology, IJCV, vol 40(2), pp: 123-148. 


\section{APPENDIX:}

\section{A1. Geometric Jacobian $\mathbf{J}(\boldsymbol{\theta})$ :}

$$
\mathbf{J}(\boldsymbol{\theta})=\left[\begin{array}{cccc}
-\mathrm{s}_{1}\left(\mathrm{l}_{1} \mathrm{c}_{2}+\mathrm{l}_{2} \mathrm{c}_{23}+\mathrm{l}_{3} \mathrm{c}_{234}\right) & -\mathrm{c}_{1}\left(\mathrm{l}_{1} \mathrm{~s}_{2}+\mathrm{l}_{2} \mathrm{~s}_{23}+\mathrm{l}_{3} \mathrm{~s}_{234}\right) & -\mathrm{c}_{1}\left(1_{2} \mathrm{~s}_{23}+\mathrm{l}_{3} \mathrm{~s}_{234}\right) & -\mathrm{l}_{3} \mathrm{c}_{1} \mathrm{~s}_{234} \\
\mathrm{c}_{1}\left(\mathrm{l}_{1} \mathrm{c}_{2}+\mathrm{l}_{2} \mathrm{c}_{23}+\mathrm{l}_{3} \mathrm{c}_{234}\right) & -\mathrm{s}_{1}\left(\mathrm{l}_{1} \mathrm{~s}_{2}+\mathrm{l}_{2} \mathrm{~s}_{23}+\mathrm{l}_{3} \mathrm{~s}_{234}\right) & -\mathrm{s}_{1}\left(1_{2} \mathrm{~s}_{23}+\mathrm{l}_{3} \mathrm{~s}_{234}\right) & -\mathrm{l}_{3} \mathrm{~s}_{1} \mathrm{~s}_{234} \\
0 & \mathrm{l}_{1} \mathrm{c}_{2}+\mathrm{l}_{2} \mathrm{c}_{23}+\mathrm{l}_{3} \mathrm{c}_{234} & \mathrm{l}_{2} \mathrm{c}_{23}+\mathrm{l}_{3} \mathrm{c}_{234} & 1_{3} \mathrm{c}_{234} \\
0 & \mathrm{~s}_{1} & \mathrm{~s}_{1} & \mathrm{~s}_{1} \\
0 & -\mathrm{c}_{1} & -\mathrm{c}_{1} & -\mathrm{c}_{1} \\
1 & 0 & 0 & 0
\end{array}\right] \begin{aligned}
& \mathrm{c}_{1}=\cos \theta_{1}, \mathrm{~s}_{1}=\sin \theta_{1}, \mathrm{c}_{2}=\cos \theta_{2}, \mathrm{~s}_{2}=\sin \theta_{2}, \\
& \mathrm{c}_{23}=\cos \left(\theta_{2}+\theta_{3}\right), \mathrm{s}_{23}=\sin \left(\theta_{2}+\theta_{3}\right), \\
& \mathrm{c}_{234}=\cos \left(\theta_{2}+\theta_{3}+\theta_{4}\right), \\
& \mathrm{s}_{234}=\sin \left(\theta_{2}+\theta_{3}+\theta_{4}\right)
\end{aligned}
$$

\section{A2. Distance estimate from single view}

$\alpha \mathbf{Z}=-\frac{\left\|\mathbf{x} \times \mathbf{x}^{\prime}\right\|}{(\mathbf{I} \bullet \mathbf{x})\left\|\mathbf{w} \times \mathbf{x}^{\prime}\right\|} ;$ where $\mathbf{u}, \mathbf{v}$ and $\mathbf{w}$ are vanishing points in $\boldsymbol{X}, \boldsymbol{Y}$ and $\boldsymbol{Z}$ directions, $\mathbf{X}$ and $\mathbf{x}^{\prime}$ are the image points whose distance are to be measured, the affine scale factor $(\alpha)$ has been determined from the known height and width of the monitor the reference plane, the vanishing line between two vanishing points $\mathbf{u}$ and $\mathbf{v}$ direction is $\overline{\mathbf{I}}$ and $Z$ is the measured distance between $\mathbf{x}$ and $\mathbf{X}^{\prime}$ [23].

\section{A3 DLS : Implemented Algorithm}

find $\Delta \theta: \min [\mathrm{J} \Delta \theta-\mathrm{x}]^{2}+\lambda^{2}[\Delta \theta]^{2}$

(where $\lambda \in \mathrm{R}$ a non zero damping constant).

$\Rightarrow$ find $\Delta \theta: \min \left\|\left(\begin{array}{c}\mathrm{J} \\ \lambda \mathrm{I}\end{array}\right) \Delta \theta-\left(\begin{array}{c}\mathrm{x} \\ 0\end{array}\right)\right\|$

$\Rightarrow \Delta \theta=\mathrm{J}^{\mathrm{T}}\left[\mathrm{J}^{\mathrm{T}} \mathrm{J}+\lambda^{2} \mathrm{I}\right]^{-1} \mathrm{x} ;$ from Normal equation

$\Rightarrow \Delta \theta=\mathbf{J}_{\text {DLS }} \mathbf{X} ;$ where $\mathbf{J}_{\mathrm{DLS}}=\mathbf{J}^{\mathrm{T}}\left[\mathrm{J}^{\mathrm{T}} \mathrm{J}+\lambda^{2} \mathrm{I}\right]^{-1} ;$

In Singular Value Decomposition (SVD) form

$\mathbf{J}_{\text {DLS }}=\mathbf{J}^{\mathrm{T}}\left[\mathrm{J}^{\mathrm{T}} \mathbf{J}+\lambda^{2} \mathrm{I}\right]^{-1}=\sum_{\mathrm{i}=1}^{\mathrm{r}} \frac{\sigma_{\mathrm{i}}}{\left(\sigma_{\mathrm{i}}+\lambda^{2}\right)} \mathrm{V}_{\mathrm{i}} \mathrm{u}_{\mathrm{i}}$

\section{\% Algorithm}

$\%$ Given initial frame $\leftarrow q_{0}$;

$\%(p \leftarrow$ position, $\phi \leftarrow$ orientation $)$;

\%Given : Current Tip trajectory $\left[T_{i}(p, \phi)\right]$;

$\% l^{0} \leftarrow$ initial fixed joint offset $\leftarrow$ curve $p_{f}$

$\% l^{i} \leftarrow$ measured joint offset $\leftarrow$ curve $p_{v}$

$\%$ Given : $l^{0}, l^{i}$

$q=q_{0} ; \%\left[\begin{array}{lllll}0 & \mathrm{pi} / 2 & 0 & 0\end{array}\right]$

$\%$ for svd formulation

$J_{0}=\operatorname{jacobian}\left(l^{0}, q_{0}\right)$;

$[U S V]=\operatorname{svd}\left(J_{0}\right)$; $\left[\begin{array}{ll}r & c\end{array}\right]=\operatorname{size}(S) ; \%$ size of the matrix for storing

$r_{\max }=\operatorname{rank}(S) ; \%$ Max. rank of s matrix.

$J_{s v d}=z \operatorname{eros}(c, r) ; \%$ initialise size of $J_{s v d}$

|for $i=1$ : no of frames $(k)$

| | while ndq > stol

| $\quad T j=T_{t} ; \%$ target

| | Ti $=$ fkine $\left(l, q_{i}\right) ; \%$ FK estimate

| $\mid d e=\operatorname{differential}\left(T_{i}, T_{j}\right)$;

$\mid \quad J_{D L S}^{i}=J_{i}^{T}\left[J_{i}^{T} J_{i}+\lambda_{i}^{2} I\right]^{-1} ;$

| | \% if through SVD then

| | \% for $i=1: r_{\max }$

| $|\quad| \% P(i)=S(i, i) * V(:, i) * U(:, i)^{\prime}$;

$|\quad| \% Q(i)=\left(S(i, i) * S(i, i)+\lambda_{i}^{2}\right)$;

| $|\quad| \% N(i)=P(i) / Q(i)$;

$|\quad| \% J_{s v d}=J_{s v d}+N(i)$;

| $\%$ end

| $\mid \quad d q=J_{D L S}^{i} * d e$;

| $\quad n d q=\operatorname{norm}(d q)$;

| $q=q+d q$;

| $\mid$ end

| $q_{i}=q$

| end 